\title{
Towards Understanding the External links of Video Sharing Sites: Measurement and Analysis
}

\author{
Kunfeng Lai \\ Department of Computing \\ The Hong Kong Polytechnic University \\ Email: cskflai@comp.polyu.edu.hk
}

\author{
Dan Wang \\ Department of Computing \\ The Hong Kong Polytechnic University \\ Email: csdwang@comp.polyu.edu.hk
}

\begin{abstract}
Recently, many video sharing sites provide external links so that their video or audio contents can be embedded into external web sites. For example, users can copy the embedded URLs of the videos of YouTube and post on their own blogs. Clearly, the purpose of such functionality is to increase the distribution of the videos and the associated advertisement. In this paper, we provide a comprehensive measurement study and analysis on these external links. With the traces collected from two major VOD sites, YouTube and Youku of China, we show that the external links have various impact on the popularity of the VOD sites. More specifically, for videos that have been uploaded for eight months in Youku, around $15 \%$ of views can come from external links. Some contents are densely linked, for example, the comedy videos can attract more than 800 external links on average. We also study the relationship between the external links and the internal links. We show that there are correlations; for example, if a video is popular itself, it will likely have a larger number of external links. Another observation is that we always find that the external links of Youku usually have a higher impact than that of YouTube. We conjecture that a more regional site may enjoy a relatively higher impact from the external links.
\end{abstract}

\section{Categories and Subject Descriptors}

H.5.1 [Multimedia Information Systems]: [Video]

\section{General Terms}

Measurement, Performance.

\section{Keywords}

VOD sites, measurement, external links

\section{INTRODUCTION}

These years have witnessed the large explosion of the popularity of the online UGC (user generated content) sites. In these sites, people are not only the information consumers, but they can also actively upload contents of their own. A common belief of their success is that through the UGC sites and the online social network

Permission to make digital or hard copies of all or part of this work for personal or classroom use is granted without fee provided that copies are not made or distributed for profit or commercial advantage and that copies bear this notice and the full citation on the first page. To copy otherwise, to republish, to post on servers or to redistribute to lists, requires prior specific permission and/or a fee.

NOSSDAV'10, June 2-4, 2010, Amsterdam, The Netherlands.

Copyright 2010 ACM 978-1-4503-0043-8/10/06 ...\$10.00.

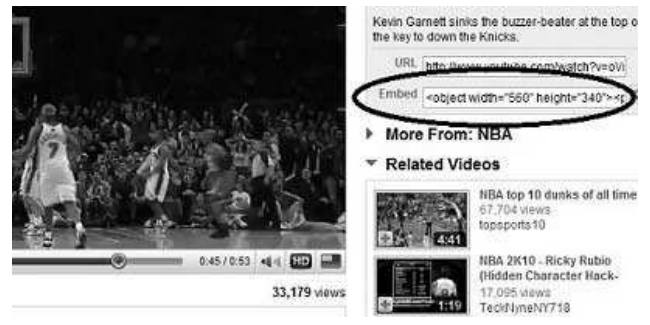

Figure 1: The external link

generated by them, the information distribution can be much faster; and in a wider scale. There are various UGC sites with different targets, e.g., Facebook for general online communities and Flickr for photo sharing sites. Among them are the VOD sites represented by YouTube [20] and Youku [21] where this paper will focus on.

These VOD sites provide numerous functionalities to expedite video distribution; for example, there are the related video links (See Fig. 7) which arrange the videos by similar topics. There are other internal links to organize the users, videos, comments within the VOD sites together. Many previous works have studied the internal interactions [7][13] in details and showed that they contribute to the spread of the popularity of the these video sharing sites.

Another important function of these VOD sites is the external links. An example of the external link is shown in Fig. 1. We can see that for each video in YouTube, an embedded link is provided. The user can copy and paste this link into anywhere such as their personal webpages, blogs, or even in forums. When people watch the videos from these external environment, traffic and click counts go through YouTube. Clearly, such function allows YouTube to penetrate into the cyber-world deeper and information distribution even faster, as now it attracts views from non-YouTube users.

In this paper, we are interested in these external links. Compared with the flourishing studies on the internal environment of the VOD sites, we are the first to concentrate on the exterior environment of these VOD sites. We have several contributions in this paper, 1) we develop a customized crawler that can collect the number of external links and the total views from the top external links. Note that tracing the external links is more difficult as it is in the opposite direction of tracing the internal links, where one can follow the internal links inductively. We find it difficult to obtain the total views from all the external links, unless the entire Internet is crawled. As such we approximate the results by a detailed analysis from the top external links we collected; 2) we conduct a comprehensive measurement on the external links of YouTube and Youku. To the best of our knowledge, there is no work on Youku and there is no work that directly comparing two similar VOD sites. We show that the impact of external links is non-trivial and there are quite substantial differences between these two VOD sites. 3) we conduct measurement on both external links and some important internal links. We 


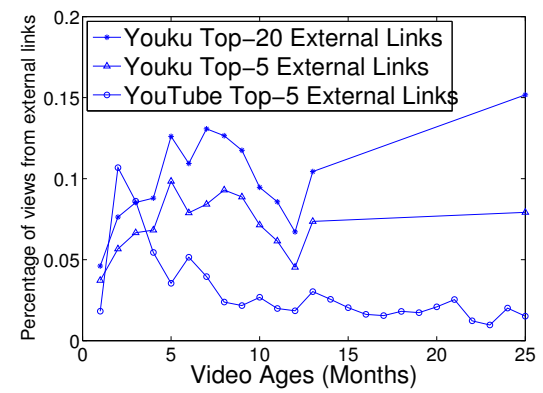

(a) External views vs. video age

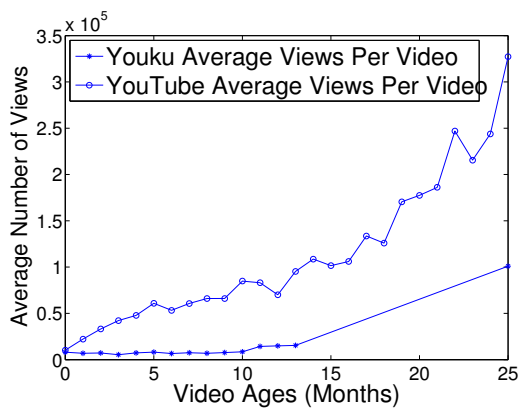

(b) Total views vs. video age

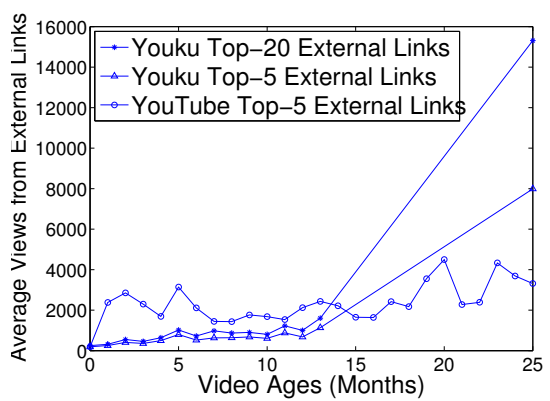

(c) External views vs. video age

Figure 2: Impact of external views on the videos

study their correlations. We illustrate that different parameters have different impact on the popularity of the videos.

The remaining part of our paper is organized as follows. Section 2 discusses the background and measurement methodology. The characteristics of the external links are presented in Section 3. Section 4 is devoted into the comparison of the correlation between internal links and external links. Section 5 lists the related work and finally, we conclude our paper in Section 6.

\section{BACKGROUND AND MEASUREMENT METHODOLOGY}

\subsection{Background and Motivation}

Currently, there is rapid growth of the web sites of the online social networks. One key feature of these web sites is that the users are not only the information consumers, but also actively upload contents of their own. One notable class of online social network web sites is for video sharing, represented by YouTube and Youku. These video sites have attracted a great number of studies in the recent years. These studies, however, focus on various internal links of the video sharing sites. That is, they work on the relationship of the users and the videos within the video sites.

To widely distribute the content videos and to attract more users, these video sites provide external links for the videos. The users can easily obtain an embedded link of a video and paste the link to any web pages in other web sites, such as forums, or their blogs. In this paper, we define the internal links as those maintaining a relationship within the web sites. These links include the user-video, user-user, video-video relationship. We define the external links as the links to the videos that are embedded in other web sites.

Though these external links are important since, we believe, they are designed to improve the popularity of the video sharing sites, to the best of our knowledge, there is no study on their characteristics. Therefore, we would like to ask 1) what the impact of the external links on the videos is; e.g., how many views are contributed by external links, and 2) what the relationship of the external links and internal links is; their differences, interactions and correlations. Such curiosities motivate this paper.

\subsection{Measurement Methodology}

Our experimental datasets come from two user generated content VOD sites, YouTube [20] and Youku [21]. YouTube is one of the largest UGC VOD sites in the world and at the time of this paper is being written, and it accepts roughly 1.886 billion views [19] every day. Youku is the most popular video site in China [19], with page views of 40.9 million per day [19].

We built a crawler to collect the necessary information. For YouTube, we started our sampling from the recent videos on Mar. 24th, 2009. We recursively crawled all the related videos for 7 days until Mar. 31st, 2009. In total, we have collected $1.24 \times 10^{6}$ videos from YouTube. For Youku, we started our sampling from the most featured videos in the main page on July 8th, 2009 and recursively crawled the related videos for 5 days. In total we have $1.43 \times 10^{6}$ videos collected. Note the emphasis here is that we have collected $1+$ million videos for each site. The upload time of the videos spans from weeks ago to years ago, not only in the 7 (or 5) day window of our data collection.

To collect the external links of YouTube, we used a universal Java Script engine provided by Google [22]. This engine can parse the Java Script codes from video pages, so as to track the external link information maintained by YouTube internally. With this engine, we could get the URLs of the external links as well as the number of views of each external link. Unfortunately, YouTube only maintains the top- 5 external links (in terms of the number of views) of each video. We have yet to find another method that can collect the information of all the external links of the videos. Intrinsically, YouTube does not provide an interface to release such information (or they may not have that information themselves). Without such interface, unless one can crawl the entire Internet, it is unlikely that all the external links can be collected. Thus, in our study, we only use the top-5 external links. For Youku, we used similar methodology. Youku provides more information, and we could get the total number of external links for each video, the URLs and the total number of views of the top-20 external links.

We admit that collecting information only from top external links affects the accuracy of the study. Our argument is that, on one hand, part of our studies focus on the evolution of the views from external links. In such situation, the results are less affected. On the other hand, for the Youku trace, we have the total number of external links, and the total number of views of the top-20 external links. Thus, we have a strong basis to analyze the remaining part of the views of external links. We did find that the external views in a large sampling space fit the power law with a $r$-square over $99.8 \%$. Therefore, we are more confident that our prediction of the total views are close to reality. The remaining part of our paper use external links to denote the top-5 external links for YouTube and top-20 external links for Youku if there is no ambiguity.

Besides the external links, we also collected the information of internal links (we focus on the related video links) for comparison study. For such data collection we adopt the same strategies as [1].

\section{CHARACTERISTICS OF THE EXTER- NAL LINKS}

\subsection{An Overview of External Links}

We first show the impact of the external views on the videos in Fig. 2. We classify the videos according to their ages, i.e., the total duration since they have been uploaded to the VOD sites. Note that YouTube provides the upload date for each video, whereas Youku provides a rougher estimation of how many days or months or years a video has been uploaded. For example, the videos uploaded 13 months or 14 months ago in Youku will all be labeled as 'uploaded one year ago'. As such, the points 13-month and 25-month in our 
figures for Youku stand for the videos uploaded one year and 2 years ago. Note that our results are not affected as the points in our figures are the average (not accumulative) number of views.

In Fig. 2 (a), we show the percentage of the views that come from external links. We see that for the videos in YouTube with an age of two months, $10 \%$ of the views come from the top-5 external links. For videos with an older age, the percentage of the views from external links gradually drops to around $2 \%$. For Youku, the impact of external links is much higher. For most of the videos, more than $8 \%$ of views are from the exterior environment. For videos with an age of 24 months, views from external links can contribute as many as $15 \%$. Even consider the top-5 external links, they contribute about $6 \%-9 \%$ of total views, which is still more significant than YouTube.

To explain the situation more clearly, we show the specific number of the total views of the videos and the total views from external links as a function of the video ages. In Fig.2 (b) we show the total views, averaged per video, for different video age groups (this includes both views from internal links and external links). Clearly, YouTube attracts much more views than Youku. The total views increase steadily for both YouTube and Youku as the video ages increase. In Fig.2 (c), we show the total views from external links (averaged per video). We see that for YouTube, the total external views are comparatively stable among all video ages groups. The external views are concentrated by the first few months. This is likely because for the external links which referee the video, their exposure will also reduce when time passes and the exposure of their posts is superseded by more recent posts, making the views to the external linked videos drops very fast. As such, there is very moderate accumulation of the external views. For Youku, the total external view increases with the time and the discrepancy of views in external view groups is not that dramatic.

We noticed that there is a big difference between the impact of the external links on YouTube and Youku; which does not conform to our expectation. We consider a possible explanation can be that YouTube, as a VOD site of world-wide popularity, the external links are widely spread to the external websites all over the world. These external websites may not be world-wide popular, however. Thus, the external links cannot obtain world-wide popularity and have less impact (in terms of percentage). Youku, on the contrary, has popularity within China only. The external links are also on the Chinese-based websites and can have China-wide popularity. As such, the comparative impact of the external links of Youku is much higher than YouTube. Based on our current data, we are unable to verify this. As a very first work on external links, we confine ourselves to the very fundamental problems such as the correctness of the data collection, and the understanding of the basic characteristics of the external links, as will be presented in the remaining part of the paper. We will leave such questions to our future work.

\subsection{The Number of External Links}

Fig. 3 plots the number of videos as a function of the number of external links in a log-log scale for different age groups. Since YouTube cannot provide the total number of links, we only study Youku in this figure. We can see clearly that a small portion of videos enjoy the majority of the external links. We therefore use power law distribution $f(x)=\alpha \times x^{-s}$ to fit the data. We find that the power law distribution fairly matches the external link distribution. This is especially true for the videos that have a large number of external links, i.e., the tails of the distribution. We find that the 'tails' follow power law with a $r$-square of more than $99 \%$ (for specific $r$ for each age group, we redirect the readers to our technical report [10] for the sake of space limitation). This is not entirely surprising. Notice that the power law behavior has been observed in various properties of the UGC. For example, it is shown that the distribution of the number of views in YouTube also fit the power law distribution [1][3].

Generally, the exponent $s$ decreases (the power low fit line moves rightwards in Fig. 3) when the video age increases. A smaller $s$ with the ages of the videos means that a larger proportion of the videos has more external links.

\subsection{The Views from the External links}

We study the views contributed by the external links. We still use the Youku data set for analysis. Fig. 4 shows the total views of the external links as a function of the ranks of the external links in a log-log scale. Note that the rank is from one to 20 as we only have the views of the top- 20 external links. We plot the data for the videos with an age of one month, seven months and 12 months in Fig. 4. We see that the number of views also matches the power law distribution for all three age groups.

Next we would like to estimate the total views from all the external links. Note that we already have the total views of the top-20 external links, and we denote it as $\mathcal{V}_{20}$. The unknown part is the views from the external links with the rank greater than 20 . We divide this set into two groups: 1) the videos with more than 20 external links and 2) the videos with less than 20 external links. For Group 2, we can easily bound the number of views. Thus, our primary target is to estimate the total views for Group 1.

In Fig. 5 we plot the total views of the external links as a function of the ranks of the external links in a log-log scale for the videos with more than 20 external links. We plot the data for the videos with an age of one month, seven months and 12 months. We can see that the number of views also matches the power law distribution. Again, to fit the power law distribution, let $\mathcal{V}(t)$ be the total number of views for videos with age $t$. Let $\mathcal{R}$ be the rank of the external links. Let $p(t)$ be an exponential factor. Let $a(t)$ be an adjustment factor. We also plot the power law distribution $\mathcal{V}(t)=a(t) \times$ $\mathcal{R}^{-p(t)}$ for different age groups in Fig. 5. We see that the videos groups fit power law distribution better (we put the parameters of the power law in our technical report [10] due to space limitation).

Such observation indicates that most external views are contributed by the top external links (i.e., high rank external links). Therefore, we conjecture that even though we only collect the top20 (or top-5) of the external links, they are representative enough. To verify this, we estimate of the total external views given that the power law distribution can roughly approximate the views.

Let $\mathcal{V}_{1}^{*}(t)$ be the total views for the videos of Group 1 .

$$
\mathcal{V}_{1}^{*}(t) \approx \sum_{r=21}^{\infty} a(t) \times r^{-p(t)} \leq a(t) \times \frac{1}{p(t)-1} \times 20^{(1-p(t))}
$$

The last inequality holds from the Cauchy convergency test if $p(t)>$ 1. We have observed that this is true in practice (see specific values of $p(t)$ in our technical report [10]). This completes the estimation of the views of Group 1. For Group 2, we obtain the accurate total views $\mathcal{V}_{2}$ provided by the video site.

Therefore, the estimated total view is $\mathcal{V}_{20}+\mathcal{V}_{1}^{*}(t)+\mathcal{V}_{2}$. We plot this number in Fig. 6. We see that the total views from the top-20 external links are very close to the total views when the video ages are less than 12 months.

\subsection{The External Links from Different Video Categories}

We have studied the characteristics of the external links. Now we take another angle and study from the point of view of the videos. We plot the number of external links of different categories in Youku in Fig. 7 (a). In this figure, the categories are ranked by the average external links on each video. We can see that the number of external links of the videos is substantial. For example, for a comedy, there can be as many as 878.1 external links. Looking into the details of our log file, we see that many comedy videos are linked by a substantial number of different users in their blogs. Some videos are linked in a great many pages in web forums. This actually suggests that external links can greatly increase the popularity of the videos. 

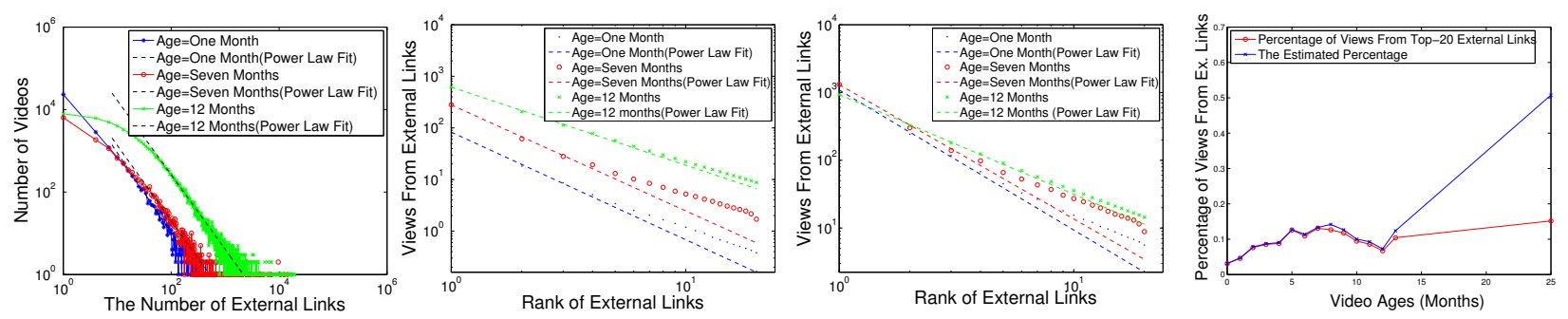

Figure 3: The No. of external Figure 4: External views vs. Figure 5: External views vs. Figure 6: The estimated Ex. links rank (all videos) rank (No. of links $\geq 20$ ) views

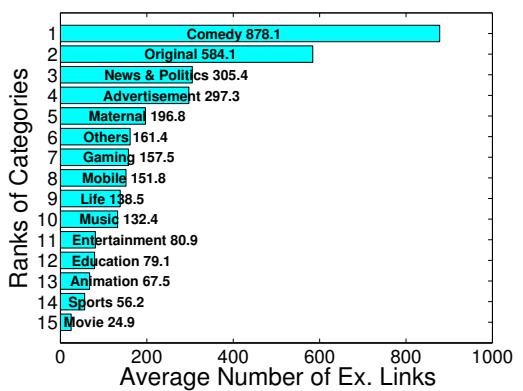

(a) The average number of external links per video

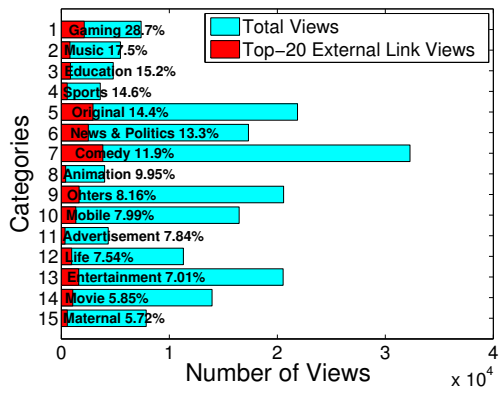

(b) Average views from top-20 external links per video (Youku)
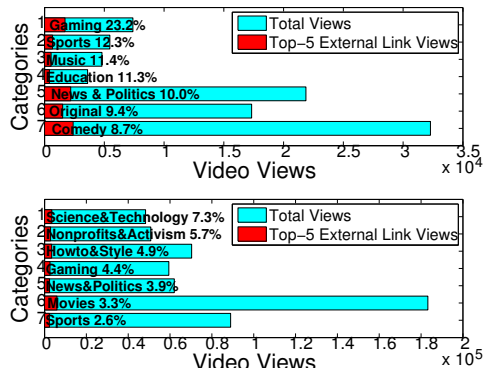

(c) Average views from top-5 external links per video

Figure 7: The impact on video categories from the external links

In Fig. 7 (b), we select 15 categories in Youku, which have the highest percentage of external views. We plot the total views of each category (in green) and the external views of the top-20 external links (in red). In the figure, "Comedy" attracts the largest number of external views, on average 3847.5 per video. This is not surprising as "Comedy" also attracts the largest number views (32286.2), representing the popularity of comedy videos in general. We also see that "Gaming" attracts 7391.4 views in total and 2125.0 views from external links. This is the highest percentage. This suggests that people would like to view "gaming" videos in their respective external environment.

To compare Youku and YouTube, we show the views of different categories based on their respective views of the top-5 external links (see Fig. 7 (c)). In general, YouTube attracts an order of magnitude more views than Youku. The percentage of external views is much smaller. This conforms to the observation from Fig. 2. Another observation is that the categories that are most viewed by external links are substantially different in YouTube and Youku. For example, they share in common only 3 out of 7 categories, namely "Gaming", "Sports", and "News and Politics". This might show the differences in the flavors between the users in different geographic regions. In addition, the more success in Youku in extending its impact into the external environment could suggest that there may be also potential for YouTube to increase its external views.

\subsection{Summary}

We summarize our major observations in this section as follows: 1) The sheer number of external views and the external links are substantial for both YouTube and Youku. The external views/links have contributed greatly to Youku while it still remains small to YouTube; 2) Most of the external links are linked to a small number of videos, i.e., the number of external links conforms to power law distribution; it fits especially well for the videos with large number of external links; 3) The number of external views also conforms to power law distribution; it fits better for old-age videos. Though we cannot obtain the total views of all the external links, with the observation of power law, we can deduct that the views from top-
20 external links is representative enough for videos which are less than 12 months old; 4) Different video categories attract different percentage of external views. In some categories, e.g., "Gaming", almost $30 \%$ of views are contributed by external links.

\section{EXTERNAL LINKS VS. INTERNAL LINKS}

To further understand the impact of external links on the UGC video sites, we study the relationship between internal links and external links which respectively represent the internal environment and the external environment. According to Alexa [19], a user spends an average of 22 minutes on YouTube and 6.7 minutes on Youku everyday. As a result, we infer that many users would watch multiple videos in the VOD sites. For these users, a most straightforward way is to follow the related video list (See Fig. 7). We call a video the parent video for the videos in the related video link list. We specifically focus on the relationship of the external links, the related video links (We call them the $R$-links thereafter) and the popularity of the parent videos on the external links. In addition, we also study the relationship between the external links and other factors, such as the total views of the video.

\subsection{Internal Parameters and External Links}

Fig. 8 presents the relationship of views with various internal factors of R-links, parent views and total views of the video. We

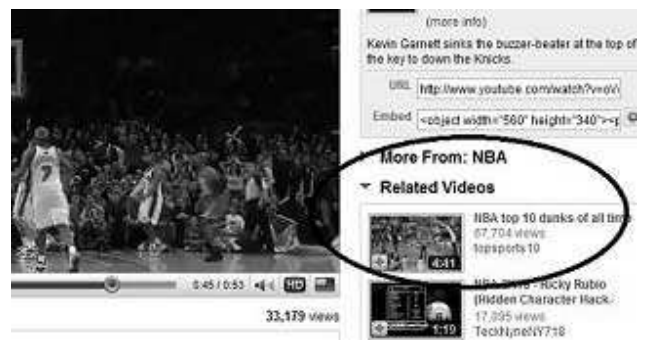

Figure 7: The related video link (R-link) 


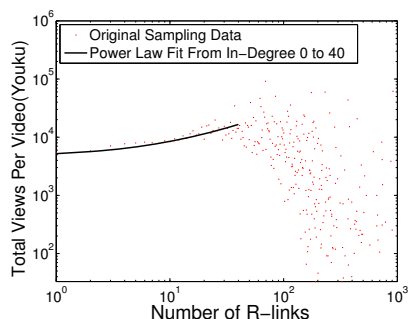

(a) The number of R-links vs. total (b) views (Youku)

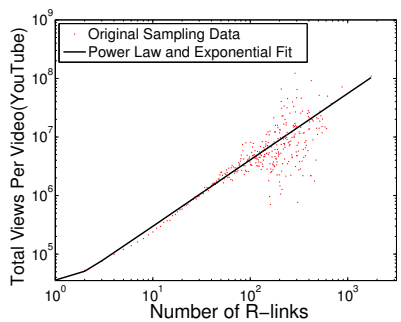

(b) The number
views (YouTube)

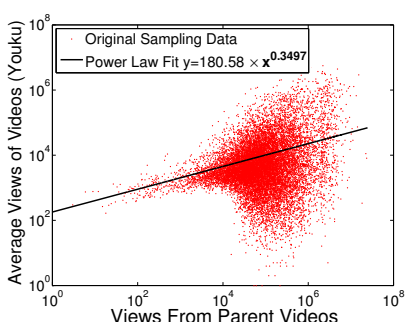

(c) Views of part

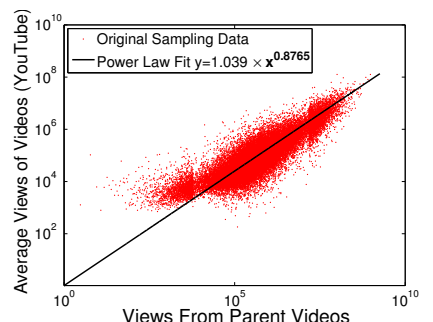

(d) Views of parent videos vs. total views (YouTube)

Figure 8: The links vs. the views

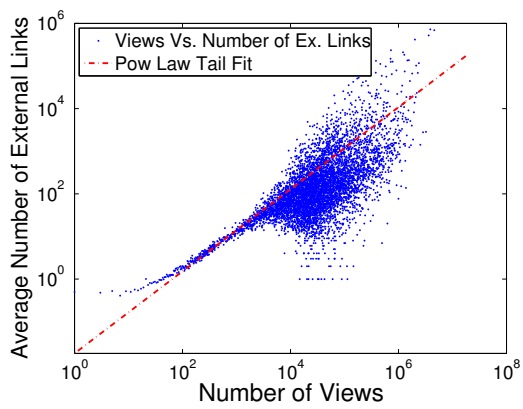

(a) Video views vs. the number of ex. links

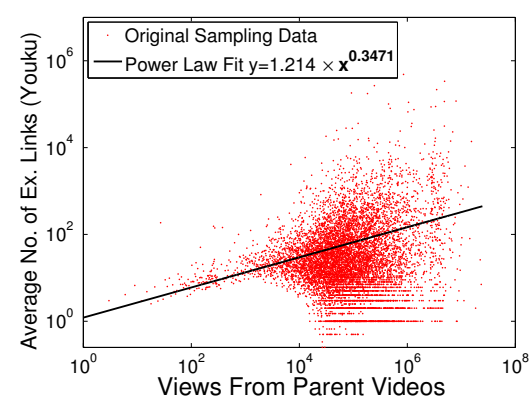

(b) Views of parent videos vs. number of external links

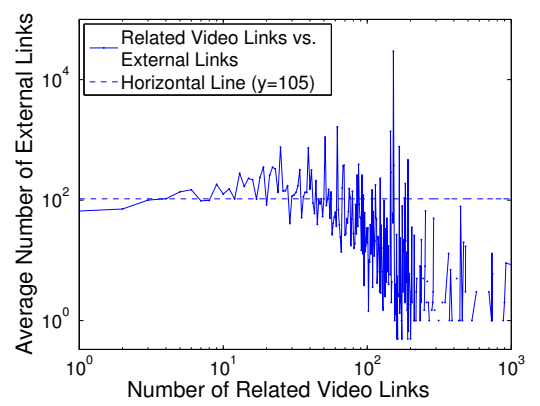

(c) The number of R-links vs. the number of ex. links

Figure 9: External links vs. internal factors (Youku)

\begin{tabular}{c|c|c|c|c}
\hline \hline Corr & views & Ex. links & R-links & parent views \\
\hline views & 1 & 0.506 & -0.018 & 0.22 \\
\hline Ex. links & 0.506 & 1 & -0.029 & 0.20 \\
\hline R-links & -0.018 & -0.029 & 1 & 0.23 \\
\hline parent views & 0.22 & 0.20 & 0.23 & 1 \\
\hline \hline
\end{tabular}

\section{Table 1: The correlation coefficient of parameters in Youku}

plot in Fig. 8 (a) the number of internal views as a function of the number of R-links of Youku. Here, we can hardly see any impact of the R-links on the improvement of the internal views of Youku. We plot in Fig. 8 (b) the number of total views as a function of the number of R-links of YouTube and we see that there is a correlation. This shows that more R-links will lead to an increase of the views of YouTube. We plot in Fig. 8 (c), (d) the average views of a video as a function of the views from the parent video for Youku and YouTube respectively. We see that in general, the popularity of the parent videos has a positive impact on the video views for both Youku and YouTube, and the parent views of YouTube shows a stronger impact.

Fig. 9 depicts the relationship between the number of external links with the three internal factors, namely the number of views, the views from parent videos and the number of R-links. All the results in Fig. 9 are from Youku data set. Fig. 9 (a) shows that there is a clear relationship between the views of videos and the number of external links. Especially when the number of views is 100 , we can see this relationship is almost linear. This can also explain the result in Section 3.2, the power law 'tail' of the number external links. Fig. 9 (b) shows the views from the parent videos has weaker correlation with the number of external links. The number of external links scatters as the views of parent videos grows. In Fig. 9 (c), we see there is even weaker relationship between the number of R-links and the number of external links in Youku.

\subsection{Analysis of the Correlation}

We next conduct an in-depth analysis on the correlation coef-

\begin{tabular}{c|c|c|c}
\hline \hline Corr & views & R-links & parent views \\
\hline views & 1 & 0.49 & 0.77 \\
\hline R-links & 0.49 & 1 & 0.60 \\
\hline parent views & 0.77 & 0.60 & 1 \\
\hline \hline
\end{tabular}

Table 2: The correlation coefficient of parameters in YouTube

ficients ${ }^{1}$ between the external link and internal factors for both Youku and YouTube (see Table 1 and Table 2). In Table 1 we find that the total views and the number of external links of Youku are most correlated with a correlation coefficient of 0.506 . The number of R-links hardly affects the number of external links and the total views. The views from parent videos weakly correlated with all the factors. This conforms to the general intuition as the popularity of the video itself will directly impact on the external links (and vice versa); the popularity of the parent video will have a more moderate impact and a larger related video links will have an even weaker impact on the external links.

Compared with Youku, the internal connections in YouTube are much tighter. In details, we can see that the relationship of the number of R-links, the parent views and the total views are high $(0.77,0.60,0.49$ respectively).

\subsection{Summary}

We summarize our observation in this subsection as follows: 1) the number of external links are mostly and more directly affected by the total views of the videos; 2) the number of external links can be affected indirectly by such internal factors as parent views and the number of R-links, since these factors can increase the views

\footnotetext{
${ }^{1}$ Here, a correlation coefficient of 1 indicates that the two parameters are linearly correlated, i.e., one parameter will increase (or decrease) linearly with the other parameter. A correlation coefficient of -1 indicates that one parameter will increase linearly as the other parameter decreases.
} 
of videos; 3) we see that the internal factors in YouTube have a stronger correlation than that in Youku.

\section{RELATED WORK}

Nowadays, online social network sites, such as Facebook, MySpace and Flickr have attracted millions of people all of the world. These sites are substantially different from the conventional sites as the users are highly involved in the content generation and daily activities of these sites. Consequently, there is an increasing number of studies on the behaviors of these sites, especially on the user behaviors and interactions. For example, the user profiles of Facebook is studied in [18], the characteristics of the users is studied in [16] and the user interactions of Flickr is studied in [17].

Many sites with partially online social behaviors are also studied, for example, Slashdot Zoo [8], Orkut [2], MSN [11], online Ecommerce [12] and a more general network [6]. There is also a flourish of interest in understanding specific features of these online social web sites. For example, in [5], the keywords generated by the users are classified; the connection of the users through tags is analyzed in [14]; and a new temporal distance metric is developed in [15] to quantify the speed of information distribution process.

There is a unique class of online social network sites which focuses on user generated videos. The most popular worldwide to date is YouTube and the largest in China is Youku. In [1] and [3] the general features of YouTube are studied. The interesting observations among the many include 1) a clear power-law distribution for the popular videos, 2) a small world phenomenon for the videos linked by internal related video links, and 3) as these sites undertake videos which have a much higher demand on the bandwidth, a peer-to-peer structure is discussed. Indeed, NetTube is developed showing the possibility of using a peer-to-peer in [4].

A comparison of different online social networks can be found in [13]; for the four online social networks studied, strong connected cores have been observed. In [7], the traffic of YouTube in campus is characterized.

In our work, we study the external links of the online social video sites for both YouTube and Youku. Clearly, the introduction of external links is to distribute information faster and more widely. It leads the video sites to penetrate deeper into the online world. Understanding the characteristics of the external links as well as their interaction with the internal links is thus important. We have a preliminary work in [9] where we presented some characteristics of the external links of YouTube. In this study, we substantially extended the measurement results and analysis of the external links. In addition, we study the correlation of the internal links. We study two similar VOD sites and we show that the two video sites have quite different characteristics in external behavior.

\section{CONCLUSION AND DISCUSSION}

In this paper, we studied in detail an important aspect of video sharing sites: the external links. The external links provide a unique way for the VOD sites to penetrate into other websites. We observed that the external links can play a non-trivial role both in terms of the number of external links on a video, and the number of views contributed to the video. We also observed that the external links have quite different impact on YouTube and Youku. We studied the external links for different video categories. We also discussed the correlations of the external links and the internal related video links. We showed that the number of internal related video links have less impact on the external links than the total views of the video. We believe that our work can provide the foundation for the VOD sites to make more targeted advertisement, customized user development, etc.

As a very first work on the external environment of VOD sites, we only concentrate on some fundamental problems, such as how the data of external links can be collected, whether the data collection on top external links only can provide a good approximation for the overall picture, and some basic aspects of the external links. There are many important problems that yet to be answered. Especially, we are interested in the cause of the big different impact of external links on Youku and YouTube where our current data set cannot provide a convincing explanation.

In the future, we can see two important directions. On the characteristics of the external links, it would be necessary to crawl more on the host websites. For example, the background of the linkage, the basic information of the post containing the external link. On the interaction of the external links and internal links, we are interested to see other internal factors. As a whole, we hope our work can foster interest in studies on external links and more innovative usage of external links to be invented.

\section{ACKNOWLEDGEMENT}

Dan Wang's work is supported by grant Hong Kong PolyU/GYG78, A-PB0R, A-PJ19, 1-ZV5W, and RGC/GRF PolyU 5305/08E. We would like to thank for our shepard Prof. Songqing Chen and the anonymous reviewers for their constructive comments which substantially improve the paper.

\section{REFERENCES}

[1] M. Cha, H. Kwak, P. Rodriguez, Y. Ahn, and S. Moon, "I Tube, You Tube, Everybody Tubes: Analyzing the World's Largest User Generated Conten Video System", In Proc. ACM IMC'07, San Diego, California, USA, October 24 - 26, 2007.

[2] W. Chen, J. Chu, and J. Luan. "Collaborative Filtering for Orkut Communities: Discovery of User Latent Behavior," In Proc. ACM WWW'09, Madrid, Spain, April 20 - 24, 2009

[3] X. Cheng, C. Dale, and J. Liu. "Statistics and Social Network of YouTube Videos", In Proc. IEEE IWQOS'08, Enschede, The Netherlands, May 28 - 30, 2008.

[4] X. Cheng and J. Liu, "NetTube: Exploring Social Networks for Peer-to-Peer Short Video Sharing", In Proc. IEEE Infocom'09, Rio de Janeiro, Brazil, April 19 - 25, 2009.

[5] A. Fuxman, P. Tsaparas, K. Achan, and R. Agrawal, "Using the Wisdom of the Crowds for Keyword Generation", In Proc. ACM WWW'08, Beijing, China, April 21 - 25, 2008.

[6] S. Goel, R. Muhamad, and D. Watts, "Social Search in "Small-World" Experiments", In Proc. ACM WWW'09, Madrid, Spain, April 20 - 24, 2009.

[7] P. , M. Arlittz, Z. Li, and A. Mahanti, "YouTube Traffic Characterization: A View From the Edge", In Proc. ACM IMC'07, San Diego, California, USA, October 24-26, 2007.

[8] J. Kunegis, A. Lommatzsch, and C. Bauckhage, "The Slashdot Zoo: Mining a Social Network with Negative Edges", In Proc. ACM WWW'09, Madrid, Spain, April 20-24, 2009.

[9] K. Lai and D. Wang "A Measurement Study of External Links of YouTube", In Proc. IEEE Globecom'09, Hawaii, USA, November 30 - December 4th, 2009.

[10] K. Lai and D. Wang "The Implication of External links on Video Sharing Sites: Measurement and Analysis", Technical Report, Department of Computing, The Hong Kong Polytechnic University, Jan, 2010.

[11] J. Leskovec, K. Lang, A. Dasgupta, and M. Mahoney, "Statistical Properties of Community Structure in Large Social and Information Networks", In Proc. ACM WWW'08, Beijing, China, April 21-25, 2008.

[12] Y. Matsuo and H. Yamamoto, "Community Gravity: Measuring Bidirectional Effects by Trust and Rating on Online," In Proc. ACM WWW'09, Madrid, Spain, April 20-24, 2009.

[13] A. Mislove, M. Marcon, K. Gummadi, P. Druschel, and B. Bhattacharjee, "Measurement and Analysis of Online Social Networks", In Proc. ACM IMC'07, San Diego, California, USA, October 24-26, 2007.

[14] S. Sen, J. Vig, and J. Riedl, "Tagommenders: Connecting Users to Items through Tags", In Proc. ACM WWW'09, Madrid, Spain, April 20-24, 2009.

[15] J. Tang, M. Musolesi, and C. Mascolo, "Temporal Distance Metrics for Social Network Analysis”, In Proc. ACM SIGCOMM WOSN'09, Bacelona, Spain, August 17th, 2009.

[16] M. Torkjazi, R. Rejaie, and W. Willinger, "Hot Today, Gone Tomorrow: On the Migration of MySpace Users", In Proc. ACM SIGCOMM WOSN'09, Bacelona, Spain, August 17th, 2009.

[17] M. Valafar, R. Rejaie, and W. Willinger, "Beyond Friendship Graphs: A Study of User Interactions in Flickr", In Proc. ACM SIGCOMM WOSN'09, Bacelona, Spain, August 17th, 2009.

[18] B. Viswanath, A. Mislove, and M. Cha "On the Evolution of User Interaction in Facebook Bimal”, In Proc. ACM SIGCOMM WOSN'09, Bacelona, Spain, August 17th, 2009.

[19] "Alexa", http://www.Alexa.com.

[20] "YouTube", http://www.youtube.com.

[21] "Youku", http://www.youku.com.

[22] "V8 - V8 JavaScript Engine", http://code.google.com/p/v8/ 